\title{
Corrections
}

\section{Correction: Huang et al., Mouse Taste Buds Use Serotonin as a Neurotransmitter}

In the article "Mouse Taste Buds Use Serotonin as a Neurotransmitter" by Yi-Jen Huang, Yutaka Maruyama, Kuo-Shyan Lu, Elizabeth Pereira, Ilya Plonsky, John E. Baur, Dianqing Wu, and Stephen D. Roper, which appeared on pages 843-847 of the January 26, 2005 issue, the authors wish to make three corrections listed below. These corrections do not affect the main conclusions and interpretations of the paper.

1. In the Results, paragraph 4, on page 845, the first line should read as follows: Biosensor responses were generally small but were enhanced significantly by incubating lingual epithelium for 30-45 min with $500 \mu \mathrm{M}$ to $1 \mathrm{mM} 5$-HTP, the immediate precursor to 5-HT, before isolating taste buds (Fig. 2, compare $C, D$ ).

2. In Figure 2D's legend on page 845, the corrected text is as follows: $D$, A procedure similar to that in $C$ was conducted on a taste bud preparation that had been pretreated with $1 \mathrm{~mm}$-hydroxytryptophan (5-HTP) to elevate the serotonin concentration in taste cells. Responses evoked by depolarization and taste stimulation are enhanced by this procedure. $\boldsymbol{E}$, Saccharin (sac), but not aspartame (aspm), evoked serotonin release from taste buds. $F$, Mianserin ( $1 \mathrm{~nm}$; shaded area) reversibly reduced the biosensor responses evoked by depolarizing taste buds repeatedly with $50 \mathrm{~mm} \mathrm{KCl}(\downarrow)$. Preparations in $\boldsymbol{E}$ and $\boldsymbol{F}$ had been pretreated with 5-HTP, as in $\boldsymbol{D}(\boldsymbol{E}, 1 \mathrm{mM}$; F, $500 \mu \mathrm{M}$ 5-HTP).

3. In Figure $3 A$ 's legend on page 846, the corrected text is as follows: $A$, Continuation of experiment illustrated in Figure $2 D$, showing sequential biosensor responses from an isolated taste bud after stimulation with $50 \mathrm{~mm} \mathrm{KCl} \mathrm{(} \downarrow$ ) or $100 \mu \mathrm{M}$ cycloheximide (cyx; $\downarrow$ ). The biosensor was tested for sensitivity at the beginning and end of the record with 3 nM 5-HT ( $\downarrow$ ). During the traces marked "Ca-free," $\mathrm{Ca}^{2+}$ in the bath $(2 \mathrm{~mm})$ was exchanged for $3 \mathrm{~mm} \mathrm{Mg}{ }^{2+}$. This and the following preparations were pretreated with $1 \mathrm{~mm}$ 5 -HTP, as described in the text.

DOI:10.1523/JNEUROSCI.1556-15.2015

\section{Correction: Yu et al., Neural Substrates of Intention-Consequence Integration and Its Impact on Reactive Punishment in Interpersonal Transgression}

For the article "Neural Substrates of Intention-Consequence Integration and Its Impact on Reactive Punishment in Interpersonal Transgression" by Hongvo Yu, Jia Li, and Xiaolin Zhou, which appeared on pages 4917-4925 of the March 25, 2015 issue, the authors wish to make four corrections listed below. These corrections do not affect the main conclusions and interpretations of the paper.

1. On page 4917, a grant number and Miss Li Zhang and Mr. Philip Blue were erroneously omitted from the acknowledgments. The correct acknowledgments are as follows: This work was supported by the Natural Science Foundation of China (Grants 91232708, 31170972) and the National Basic Research Program of China (973 Program: 2010CB833904, 2015CB856400). We thank Xirui Cui, Huihui Zheng, Yunlu Yin, Li Zhang, and Dr. Li Hu for their assistance in data collection and data analysis; and Dr. Micheal Treadway, Mr. Philip Blue, and two anonymous reviewers for their helpful comments on previous versions of this manuscript.

2. On page 4917, in the first paragraph of the Introduction, fourth line, a reference is missing. The corrected sentence is as follows: . . when evaluating the actions of others and forming reactive attitudes (Strawson, 2008) or behaviors ...

3. In Figure $1 C$ 's legend, the corrected text is as follows: In the fMRI experiment, the punishment amount in the Harm_No-harm (i.e., failed-attempt) condition positively correlated with the trait revenge score.

4. In the last paragraph of the Results, second column on page 4922, the first sentence should read as follows: The individual differences analysis revealed that the activation in the $\mathrm{rAI}$ in the accidental condition negatively correlated with trait forgiveness $(r=-0.32, p=$ 0.068; Fig. $3 C)$, while the activation in the rTPJ in the failed-attempt condition positively correlated with trait revenge $(r=0.38, p=$ 0.029; Fig. 3D).

DOI:10.1523/JNEUROSCI.1511-15.2015 